\title{
The Representation of Lattice Quadrature Rules as Multiple Sums
}

\author{
By Ian H. Sloan and James N. Lyness*
}

\begin{abstract}
We provide a classification of lattice rules. Applying elementary group theory, we assign to each $s$-dimensional lattice rule a rank $m$ and a set of positive integer invariants $n_{1}, n_{2}, \ldots, n_{s}$. The number $\nu(Q)$ of abscissas required by the rule is the product $n_{1} n_{2} \cdots n_{s}$, and the rule may be expressed in a canonical form with $m$ independent summations. Under this classification an $N$-point number-theoretic rule in the sense of Korobov and Conroy is a rank $m=1$ rule having invariants $N, 1,1, \ldots, 1$, and the product trapezoidal rule using $n^{s}$ points is a rank $m=s$ rule having invariants $n, n, \ldots, n$. Besides providing a canonical form, we give some of the properties of copy rules and of projections into lower dimensions.
\end{abstract}

1. Introduction. In previous papers, Sloan [11], Sloan and Kachoyan [12], lattice rules for numerical quadrature over the $s$-dimensional unit cube were introduced and defined.

A lattice rule employs as abscissas all points on an infinite lattice that lie within and on the boundary of the unit cube. Those within are assigned equal weight, and those on the boundary are assigned an appropriately reduced weight. Proper definitions and background material are presented in Section 2. There it is shown that the form

$$
Q f=\frac{1}{n_{1} n_{2} \cdots n_{t}} \sum_{j_{1}=1}^{n_{1}} \cdots \sum_{j_{t}=1}^{n_{t}} \bar{f}\left(\frac{j_{1} \mathbf{z}_{1}}{n_{1}}+\cdots+\frac{j_{t} \mathbf{z}_{t}}{n_{t}}\right),
$$

where $\bar{f}$ is a periodic extension of $f$, and $\mathbf{z}_{1}, \mathbf{z}_{2}, \ldots, \mathbf{z}_{t}$ are vectors with integer components, is a lattice rule. The expression (1.1) is computationally convenient, but far from unique. This lack of uniqueness hinders computer searches for lattice rules with appropriate qualities. In this paper we seek representations of lattice rules in which at least the numbers $t, n_{1}, n_{2}, \ldots, n_{t}$ are uniquely determined; the vectors $\mathbf{z}_{1}, \ldots, \mathbf{z}_{t}$ remain nonunique. First, however, we need to gain greater insight into the structure of lattice rules.

To this end we employ elementary group theory. The concepts we require are collected together in Section 3. The connection with group theory is apparent, in that under appropriate circumstances the $i$ th summation in (1.1) corresponds to a cyclic group of order $n_{i}$, and the set of abscissas $A(Q)$ is the direct sum of $t$ such cyclic groups.

Received August 25, 1987; revised June 13, 1988.

1980 Mathematics Subject Classification (1985 Revision). Primary 65D30, 65D32.

*This work was supported in part by the Applied Mathematical Sciences subprogram of the Office of Energy Research, U.S. Department of Energy, under contract W-31-109-Eng-38. 
In Section 4 this theory is exploited to classify lattice rules. As a result, we are able to show that every lattice rule can be written in the form (1.1). Moreover, we are able to ascribe to any lattice rule a unique rank $m$ and a unique set of invariants $n_{1}, n_{2}, \ldots, n_{s}$ - the rank being the least value of $t$ in an expression of the form (1.1). Our standard form of the lattice rule uses the $m$ leading invariants $n_{1}, n_{2}, \ldots, n_{m}$, together with suitable integer vectors $\mathbf{z}_{1}, \mathbf{z}_{2}, \ldots, \mathbf{z}_{m}$, in the expression (1.1) with $t$ set equal to $m$. The remaining invariants $n_{m+1}, \ldots, n_{s}$ all have the trivial value 1 .

Using this classification, the 'number-theoretic' rules of Korobov [8], Hlawka [4], Conroy [1], Zaremba [13], Keast [7], Niederreiter [10], and Hua and Wang [5], which are of the form

$$
Q f=\frac{1}{N} \sum_{j=1}^{N} \bar{f}\left(\frac{j \mathbf{z}}{N}\right),
$$

are rules of rank 1 and invariants $N, 1,1, \ldots, 1$; and the $s$-dimensional product trapezoidal rule

$$
T_{s}^{(n)} f=\frac{1}{n^{s}} \sum_{j_{1}=0}^{n-1} \cdots \sum_{j_{s}=0}^{n-1} \bar{f}\left(\frac{\left(j_{1}, \ldots, j_{s}\right)}{n}\right)
$$

is a rule of rank $s$ and invariants $n, n, \ldots, n$.

Section 5 explores the structure of rules obtained by the projection of lattice rules onto lower-dimensional unit cubes. Section 6 discusses $n^{s}$-copy rules. Among other things, we show that a lattice rule is of rank $s$ if and only if it is an $n^{s}$ copy of a lattice rule with smaller rank.

The scope of this paper is confined to the theory of the structure of lattice rules. Examples are chosen simply to illustrate this theory. This forms a preliminary to the important task of determining rules for use in practice; that problem will be taken up in a later paper.

2. Lattice Rules. We denote the integral to be evaluated by

$$
I f=\int_{C^{s}} f(x) d x
$$

where $C^{s}$ is the closed $s$-dimensional unit cube

$$
C^{s}=\left\{\left(x^{1}, \ldots, x^{s}\right): 0 \leq x^{i} \leq 1, i=1,2, \ldots, s\right\} .
$$

When $x$ is a real number, its fractional part is conventionally denoted by $\{x\}$. We extend this notation to vectors.

Definition. $\{\boldsymbol{x}\}$ is a vector, each of whose components is the fractional part of the corresponding component of $\boldsymbol{x}$. Thus $\{\boldsymbol{x}\}$ lies in the half-open cube $U^{s}$,

$$
U^{s}=\left\{\left(x^{1}, \ldots, x^{s}\right): 0 \leq x^{i}<1, i=1,2, \ldots, s\right\} .
$$

It is convenient to define $\bar{f}(\boldsymbol{x})$, a periodic continuation of $f(x)$ outside the unit cube. Thus $\bar{f}(\boldsymbol{x})=\bar{f}(\{\boldsymbol{x}\})$ for all $\boldsymbol{x}$, and

$$
\bar{f}(\boldsymbol{x})=f(\{\boldsymbol{x}\}), \quad\left\{x^{j}\right\} \neq 0, j=1,2, \ldots, s,
$$

so that $\bar{f}$ coincides with $f$ in the interior of the unit cube. At points $x=$ $\left(x^{1}, x^{2}, \ldots, x^{s}\right)$ on the boundary of the unit cube, $\bar{f}$ is generally not continuous. 
At such points we define

$$
\bar{f}\left(x^{1}, x^{2}, \ldots, x^{s}\right)=\frac{1}{2^{s}} \lim _{\varepsilon \rightarrow 0+} \sum_{r_{1}} \sum_{r_{2}} \cdots \sum_{r_{s}} \bar{f}\left(x^{1}+r_{1} \varepsilon, x^{2}+r_{2} \varepsilon, \ldots, x^{s}+r_{s} \varepsilon\right)
$$

with each $r_{i}$ taking only the values of +1 and -1 . If $f$ is continuous on $C^{s}$ then this limit exists and is a symmetrical average of the values of $f$ at corresponding points on opposite faces of the boundary. Thus, in the one-dimensional case,

$$
\bar{f}(1)=\bar{f}(0)=\frac{1}{2}(f(0)+f(1)) .
$$

We now introduce the notion of a lattice. The simplest case is given by:

Definition. The s-dimensional integer lattice is the set

$$
Z^{s}=\left\{\left(i_{1}, i_{2}, \ldots, i_{s}\right): i_{j} \in Z, j=1,2, \ldots, s\right\}
$$

where $Z$ is the set of integers. In general:

Definition. A lattice $L$ is an infinite set of points in $R^{s}$ with the following two properties:

1. If $x$ and $x^{\prime}$ belong to $L$ then so do $x \pm x^{\prime}$.

2. Each lattice point is the center of a sphere of finite radius containing no other point of the lattice.

We are interested particularly in the following special class of lattices.

Definition. A multiple-integration lattice is a lattice which contains the integer lattice $Z^{s}$ as a sublattice.

We are now in a position to define a lattice rule.

Definition (Sloan and Kachoyan [12]). A lattice rule $Q f$ is an approximation to If of the form

$$
Q f=\frac{1}{N} \sum_{j=1}^{N} \bar{f}\left(x_{j}\right)
$$

where $x_{1}, x_{2}, \ldots, x_{N}$ are all the distinct points of a multiple-integration lattice which lie in the half-open unit cube $U^{s}$.

Definition. The abscissa set $A(Q)$ of a lattice rule $Q$ is the set of quadrature points $\left\{x_{1}, \ldots, x_{N}\right\}$ in the half-open cube $U^{s}$.

Note that in (2.8) it is possible to replace $\boldsymbol{x}_{j}$ by $\boldsymbol{x}_{j}+\mathbf{z}_{j}$ where $\mathbf{z}_{j} \in Z^{s}$. This is because $\bar{f}\left(\boldsymbol{x}_{j}\right)=\bar{f}\left(\boldsymbol{x}_{j}+\mathbf{z}_{j}\right)$. In the definition of the abscissa set, we have taken care to remove this ambiguity.

Thus, to every lattice rule $Q$ there corresponds a multiple-integration lattice $L$, and the abscissa set of the rule is

$$
A(Q)=L \cap U^{s} .
$$

The multiple-integration lattice corresponding to the number-theoretic rule

$$
Q f=\frac{1}{N} \sum_{j=1}^{N} \bar{f}(j \mathbf{z} / N)
$$

may be expressed in many forms, including

$$
L=\{j \mathbf{z} / N: j=1,2, \ldots, N\}+Z^{s}
$$


and the abscissa set is

$$
A(Q)=\{\{j \mathbf{z} / N\}: j=0,1, \ldots, N-1\} .
$$

It may be noted that the arguments of $\bar{f}$ in the form (2.10) do not necessarily lie in $U^{s}$, and therefore may differ from elements of $A(Q)$ by integer vectors. As noted already, this is permitted by the periodic nature of $\bar{f}$. For the same reason, it is merely a matter of taste whether the summation label in (2.10) runs from 1 to $N$, or say from 0 to $N-1$.

The only $N$-point one-dimensional lattice rule is the trapezoidal rule

$$
T_{1}^{(N)} f=\frac{1}{N} \sum_{j=1}^{N} \bar{f}(j / N)=\frac{1}{N}\left(\frac{1}{2} f(0)+\sum_{j=1}^{N-1} f(j / N)+\frac{1}{2} f(1)\right) .
$$

Note that the abscissa set $A\left(T_{1}^{(N)}\right)=\{j / N: j=0,1, \ldots, N-1\}$ provides the abscissas of $\bar{f}$, but that each function evaluation of $\bar{f}$ may involve more than one function evaluation of $f$. Thus what we have termed the $N$-point trapezoidal rule (also called the $N$-panel trapezoidal rule) requires $N+1$ function values of $f$.

We shall make frequent reference to the s-dimensional product-trapezoidal rule:

$$
\begin{aligned}
T_{s}^{(n)} f & =\frac{1}{n^{s}} \sum_{j_{1}=1}^{n} \sum_{j_{2}=1}^{n} \cdots \sum_{j_{s}=1}^{n} \bar{f}\left(\frac{\left(j_{1}, j_{2}, \ldots, j_{s}\right)}{n}\right) \\
& =\frac{1}{n^{s}} \sum_{j_{1}=1}^{n} \sum_{j_{2}=1}^{n} \cdots \sum_{j_{s}=1}^{n} \bar{f}\left(\frac{j_{1} e_{1}}{n}+\frac{j_{2} e_{2}}{n}+\cdots+\frac{j_{s} e_{s}}{n}\right),
\end{aligned}
$$

where

$$
e_{j}=\left(\delta_{j, 1}, \delta_{j, 2}, \ldots, \delta_{j, s}\right)=(0,0, \ldots, 0,1,0, \ldots, 0)
$$

is a unit vector having unit component in the $x^{j}$ direction and zero components otherwise. This lattice rule employs the lattice

$$
L=\left\{\left(j_{1} / n, j_{2} / n, \ldots, j_{s} / n\right): j_{i} \in Z, i=1,2, \ldots, s\right\} .
$$

It is one of the oldest and simplest $s$-dimensional quadrature rules, but is seldom used because of its prohibitive cost in terms of function evaluations.

A complicating feature of the theory of lattice rules is that the same rule may be expressed in many different ways: for example, the two-dimensional product of the 4-point and 3-point trapezoidal rules may be written as

$$
Q f=\frac{1}{12} \sum_{j_{1}=1}^{4} \sum_{j_{2}=1}^{3} \bar{f}\left(j_{1} \frac{(1,0)}{4}+j_{2} \frac{(0,1)}{3}\right)
$$

or as

$$
Q f=\frac{1}{12} \sum_{j=1}^{12} \bar{f}\left(j \frac{(3,4)}{12}\right)
$$

And the following four forms all represent the same five-point two-dimensional rule:

$$
\begin{aligned}
Q f & =\frac{1}{5} \sum_{j=1}^{5} \bar{f}\left(j \frac{(1,2)}{5}\right)=\frac{1}{5} \sum_{j=1}^{5} \bar{f}\left(j \frac{(2,4)}{5}\right) \\
& =\frac{1}{10} \sum_{j=1}^{10} \bar{f}\left(j \frac{(2,4)}{10}\right)=\frac{1}{25} \sum_{j_{1}=1}^{5} \sum_{j_{2}=1}^{5} \bar{f}\left(j_{1} \frac{(1,2)}{5}+j_{2} \frac{(3,1)}{5}\right) .
\end{aligned}
$$


Because of all this diversity, it is useful to study underlying properties of the lattice rules. First we treat an undisciplined form of the lattice rule.

THEOREM 2.1. Any expression of the form

$$
Q f=\frac{1}{n_{1} n_{2} \cdots n_{t}} \sum_{j_{1}=1}^{n_{1}} \sum_{j_{2}=1}^{n_{2}} \cdots \sum_{j_{t}=1}^{n_{t}} \bar{f}\left(\frac{j_{1} \mathbf{z}_{1}}{n_{1}}+\frac{j_{2} \mathbf{z}_{2}}{n_{2}}+\cdots+\frac{j_{t} \mathbf{z}_{t}}{n_{t}}\right),
$$

where $\bar{f}(\boldsymbol{x})$ is the periodic continuation of $f(\boldsymbol{x})$ defined above and $\mathbf{z}_{i}$ belongs to the integer lattice $Z^{s}$, is a lattice rule.

Proof. First we note that the infinite set

$$
\left\{\frac{j_{1} \mathbf{z}_{1}}{n_{1}}+\frac{j_{2} \mathbf{z}_{2}}{n_{2}}+\cdots+\frac{j_{t} \mathbf{z}_{t}}{n_{t}}+\sum_{i=1}^{s} k_{i} e_{i}: j_{i}, k_{i} \in Z\right\}
$$

is a multiple-integration lattice $L$, but that the same point may occur for more than one choice of the indices. The abscissa set $A(Q)$ corresponding to this lattice $L$ is

$$
\left\{\left\{\frac{j_{1} \mathbf{z}_{1}}{n_{1}}+\frac{j_{2} \mathbf{z}_{2}}{n_{2}}+\cdots+\frac{j_{t} \mathbf{z}_{t}}{n_{t}}\right\}: j_{i}=1,2, \ldots, n_{i}, i=1,2, \ldots, t\right\},
$$

though again each point may appear more than once. It is straightforward to show that if the zero point 0 is repeated precisely $k$ times in $(2.21)$, then each point is repeated precisely $k$ times. Thus, the rule $Q$ is an equal weight rule, and is therefore precisely the lattice rule associated with the lattice $L$.

Note that there are virtually no restrictions in the form (2.19). The $\mathbf{z}_{i}$ may or may not be linearly dependent. The value of $t$ may exceed $s$. The components of $\mathbf{z}_{i}$ may or may not have a factor in common with $n_{i}$. In any event, the abscissa set $A(Q)$ of this rule comprises only the distinct elements of the set in (2.21).

It is convenient to introduce some further definitions.

Definition. The order $\nu(Q)$ of the abscissa set $A(Q)$ is the number of function values of $\bar{f}$ required by $Q$.

Definition. The form (2.19) for $Q f$ is termed nonrepetitive if each abscissa occurs only once.

Definition. The form (2.19) for $Q f$ is termed $k$-repetitive, or simply repetitive, if each abscissa occurs precisely $k$ times and $k>1$.

If the form (2.19) is $k$-repetitive then $\nu(Q)=n_{1} n_{2} \cdots n_{t} / k$. If it is nonrepetitive then $\nu(Q)=n_{1} n_{2} \cdots n_{t}$. Later we shall use the following straightforward lemma.

LEMMA 2.2. When the form (2.19) is nonrepetitive, the equation

$$
\left\{\frac{j_{1} \mathbf{z}_{1}}{n_{1}}+\frac{j_{2} \mathbf{z}_{2}}{n_{2}}+\cdots+\frac{j_{t} \mathbf{z}_{t}}{n_{t}}\right\}=\mathbf{0}
$$

holds for integers $j_{1}, j_{2}, \ldots, j_{t}$ if and only if $j_{i}$ is a multiple of $n_{i}$ for all $i$.

The following result about the elements of the abscissa set is of fundamental importance to our study of lattice rules.

THEOREM 2.3. The elements $x_{1}, x_{2}, \ldots, x_{N}$ of the abscissa set $A(Q)$ of a lattice rule $Q$ form an abelian group under the group operation defined by

$$
\boldsymbol{x}_{i} \text { plus } \boldsymbol{x}_{j}=\left\{\boldsymbol{x}_{i}+\boldsymbol{x}_{j}\right\}
$$


The proof is trivial and is not given here. We note that the identity element is $\mathbf{0}$ and that the inverse of $x_{i} \in A(Q)$ is $\left\{-x_{i}\right\}$. In the sequel we shall not distinguish between the set $A(Q)$ and the group $A(Q)$, and we shall use the + symbol both in its conventional sense and as the group operation symbol.

For example, in (2.17) it is clear that the abscissas $x_{j}=\{j(3,4) / 12\}, j=$ $1,2, \ldots, 12$, form a cyclic group of order 12 , generated by $(3,4) / 12$. In the form (2.16), that cyclic group of order 12 is expressed as a direct sum of cyclic groups of orders 4 and 3 . That is, defining

$$
\mathbf{c}_{1}=\frac{(1,0)}{4}, \quad \mathbf{c}_{2}=\frac{(0,1)}{3},
$$

$\mathbf{c}_{1}$ generates a cyclic group $C_{1}$ of order $n_{1}=4$ and $\mathbf{c}_{2}$ a cyclic group $C_{2}$ of order $n_{2}=3$, these two groups having only the zero element $(0,0)$ in common; and the elements of $A(Q)$ are each expressible uniquely in the form $\boldsymbol{x}_{j_{1}}+\boldsymbol{x}_{j_{2}}$ where $\boldsymbol{x}_{j_{1}} \in C_{1}$ and $\boldsymbol{x}_{j_{2}} \in C_{2}$.

In the next section we shall collect together a selection of results from elementary group theory, which we shall use in Section 4 to construct a classification of lattice rules.

3. Finite Abelian Groups. Many of the results of this paper depend critically on the structure of the group $A(Q)$. In particular, decompositions of $A(Q)$ into the direct sum of cyclic groups play a key role. The results stated here are standard results from the elementary theory of finite abelian groups, or are minor corollaries of such results. Here we simply provide a brief summary. At the end of the section we provide an example illustrating many of the concepts.

The central result is:

THEOREM 3.1. A finite abelian group $G$ may be expressed (uniquely up to an isomorphism) in the form

$$
G=D_{1} \oplus D_{2} \oplus \cdots \oplus D_{m}
$$

where $D_{j}$ is a cyclic group of order $n_{j}>1$, and

$$
n_{j+1} \mid n_{j} \quad \text { for } j=1, \ldots, m-1 \text {. }
$$

In particular, the numbers $m$ (the 'rank' of $G$ ) and $n_{1}, n_{2}, \ldots, n_{m}$ (the 'invariants') are uniquely determined.

Note that the product of the invariants is the order of the group. The theorem is proved, for example, in Ledermann [9, Section 4.5, Theorem 4]. In essence, the result is obtained by first representing $G$ as a direct sum of cyclic subgroups, each of prime-power order, and then recombining, so that for a given prime $p$ each cyclic subgroup whose order is a power of $p$ is incorporated into a different one of $D_{1}, D_{2}, \ldots, D_{m}$, starting at the left with the subgroup with the highest power of $p$. Note that we may define the rank directly by:

Definition. The rank $m$ of a finite abelian group $G$ is the smallest number of cyclic subgroups into which $G$ may be decomposed. 
Similar considerations lead to:

THEOREM 3.2. Given a direct sum decomposition

$$
G=E_{1} \oplus E_{2} \oplus \cdots \oplus E_{t}
$$

into cyclic groups $E_{i}$ of orders $n_{i}$ respectively, the group $G$ is of rank $m=t$ if and only if $n_{1}, n_{2}, \ldots, n_{t}$ have a nontrivial common factor.

We shall also find occasion to use the following theorem, which relates the rank and invariants of a subgroup to the corresponding quantities for the group itself.

THEOREM 3.3. Let $G$, a finite abelian group with rank $m$ and invariants $n_{1}$, $n_{2}, \ldots, n_{m}$, have a subgroup $G^{\prime}$ with rank $m^{\prime}$ and invariants $n_{1}^{\prime}, n_{2}^{\prime}, \ldots, n_{m^{\prime}}^{\prime}$. Then $m \geq m^{\prime}$, and $n_{i}^{\prime} \mid n_{i}$ for $i=1, \ldots, m^{\prime}$.

Proof. The result for the case in which $G$ is of prime-power order is given in Hall [3, Theorem 3.3.3]. The general result then follows by expressing $G$ and $G^{\prime}$ as direct sums of Sylow subgroups.

Next, we state a corresponding result for factor groups of $G$. (Recall that if $H$ is a subgroup of the abelian group $G$, then the factor group $G / H$ is the group formed by the 'cosets' $x+H$, with $x \in G$. The identity in $G / H$ is $H$. Loosely, the factor group $G / H$ is the group obtained from $G$ by treating as equivalent any elements of $G$ that differ by an element of $H$.)

THEOREM 3.4. Let $H$ be a subgroup of a finite abelian group $G$ having rank $m$ and invariants $n_{1}, n_{2}, \ldots, n_{m}$, and let $G / H$ have rank $m^{\prime}$ and invariants $n_{1}^{\prime}, n_{2}^{\prime}, \ldots$, $n_{m^{\prime}}^{\prime}$. Then $m \geq m^{\prime}$, and $n_{i}^{\prime} \mid n_{i}$ for $i=1, \ldots, m^{\prime}$.

Proof. Because $G$ is a finite abelian group, $G / H$ is isomorphic to some subgroup of $G$ (see Hungerford [6, p. 82, Ex. 14]). Thus the result follows immediately from Theorem 3.3.

We conclude the section with an example of a particular group of order 12,

$$
\begin{gathered}
G=\left\{(0,0,0),\left(\frac{5}{6}, 0, \frac{1}{3}\right),\left(\frac{2}{3}, 0, \frac{2}{3}\right),\left(\frac{1}{2}, 0,0\right),\left(\frac{1}{3}, 0, \frac{1}{3}\right),\right. \\
\left(\frac{1}{6}, 0, \frac{2}{3}\right),\left(\frac{2}{3}, \frac{1}{2}, \frac{1}{6}\right),\left(\frac{1}{2}, \frac{1}{2}, \frac{1}{2}\right),\left(\frac{1}{3}, \frac{1}{2}, \frac{5}{6}\right), \\
\left.\left(\frac{1}{6}, \frac{1}{2}, \frac{1}{6}\right),\left(0, \frac{1}{2}, \frac{1}{2}\right),\left(\frac{5}{6}, \frac{1}{2}, \frac{5}{6}\right)\right\}
\end{gathered}
$$

with addition modulo 1 . This group is the abscissa set for a lattice rule $Q$ with lattice

$$
L=\left\{j_{1}\left(\frac{5}{6}, 0, \frac{1}{3}\right)+j_{2}\left(\frac{2}{3}, \frac{1}{2}, \frac{1}{6}\right)+Z^{3}: j_{1}, j_{2} \in Z\right\} .
$$

A direct sum representation of $G$ in the manner of Theorem 3.1 is

$$
G=D_{1} \oplus D_{2},
$$

where

$$
D_{1}=\left\{j\left(\frac{1}{6}, \frac{1}{2}, \frac{1}{6}\right): j=0,1, \ldots, 5\right\}
$$


the cyclic group of order 6 generated by $\left(\frac{1}{6}, \frac{1}{2}, \frac{1}{6}\right)$, and

$$
D_{2}=\left\{(0,0,0),\left(0, \frac{1}{2}, \frac{1}{2}\right)\right\} \text {. }
$$

The invariants of $G$ (that is, the orders of $D_{1}, D_{2}$ ) are 6,2 ; note that, as required, the second divides the first.

To illustrate the properties of the factor group $G / H$, we take as $H$ the order 2 subgroup

$$
H=\left\{(0,0,0),\left(\frac{1}{2}, 0,0\right)\right\} .
$$

Each element of $G / H$ is a coset of the form

$$
x+H=\left\{x+(0,0,0), x+\left(\frac{1}{2}, 0,0\right)\right\},
$$

where $x$ is an element of $G$. It will be found that the six cosets form a cyclic group, generated by the element

$$
\left\{\left(\frac{1}{6}, \frac{1}{2}, \frac{1}{6}\right),\left(\frac{2}{3}, \frac{1}{2}, \frac{1}{6}\right)\right\} .
$$

Thus, this particular factor group has rank 1 and sole invariant 6 , in conformity (since the rank does not exceed 2, and since 6 divides 6 ) with Theorem 3.4 .

4. Classification of Lattice Rules. In this section we employ the results of Section 3 to classify lattice rules.

Given a lattice rule $Q$, the abelian group formed by the abscissa set $A(Q)$ may be expressed (usually in many different ways) as a direct sum of cyclic groups,

$$
A(Q)=C_{1} \oplus C_{2} \oplus \cdots \oplus C_{t} .
$$

Corresponding to this we have:

THEOREM 4.1. A lattice rule $Q$ is expressible as a nonrepetitive form

$$
Q f=\frac{1}{n_{1} n_{2} \cdots n_{t}} \sum_{j_{1}=1}^{n_{1}} \sum_{j_{2}=1}^{n_{2}} \cdots \sum_{j_{t}=1}^{n_{t}} \bar{f}\left(j_{1} \frac{\mathbf{z}_{1}}{n_{1}}+j_{2} \frac{\mathbf{z}_{2}}{n_{2}}+\cdots+j_{t} \frac{\mathbf{z}_{t}}{n_{t}}\right) .
$$

Proof. If the cyclic group $C_{i}$ in (4.1) is of order $n_{i}$ then $C_{i}$ has at least one element of that order; we call one such element $\mathbf{c}_{i}$ and choose $\mathbf{z}_{i}=n_{i} \mathbf{c}_{i}$.

Comment. Clearly, $\nu(Q)=n_{1} n_{2} \cdots n_{t}$. This theorem is a converse of Theorem 2.1 (which states that any $Q f$ of form (4.2) is a lattice rule). We refer to (4.2) as a $t$-cycle form of the rule $Q$.

Conversely, a lattice rule $Q$ given in nonrepetitive form (4.2) yields immediately a direct sum decomposition of the abscissa set (4.1), the group $C_{i}$ in (4.1) being generated by $\mathbf{z}_{i} / n_{i}$.

The 1-1 correspondence between (4.1) and (4.2) allows us to transcribe many of the results about group decomposition in Section 3 to results about nonrepetitive representations of $Q$ of form (4.2). The definitions of rank and invariants are also applied to lattice rules $Q$. Thus we have:

Definition. The rank $m$ of a lattice rule $Q$ is the least possible value of $t$ for which $A(Q)$ may be expressed in form (4.1) or $Q f$ in form (4.2). 
By extension, the trivial one-point lattice rule $T_{s}^{(1)}$, for which the abscissa set is $A\left(T_{s}^{(1)}\right)=\{0\}$, will be said to have rank 0 .

Definition. The form (4.2) of a lattice rule $Q$ is said to be minimal if $t=m$, where $m$ is the rank of $Q$.

It is useful to be able to determine whether or not a given expression of a lattice rule is minimal. This turns out to be straightforward if the form is nonrepetitive. Theorem 3.2 gives immediately:

THEOREM 4.2. A nonrepetitive form (4.2) is minimal if and only if $n_{1}, n_{2}, \ldots$, $n_{t}$ have a nontrivial common factor.

The following is a useful property of a nonrepetitive minimal form of a lattice rule.

THEOREM 4.3. If the form (4.2) is nonrepetitive and minimal (implying $t=$ $m$ ), then the vectors $\mathbf{z}_{1}, \mathbf{z}_{2}, \ldots, \mathbf{z}_{m}$ are linearly independent (with respect to the rational field).

Proof. Obviously it is sufficient to establish the linear independence of the vectors $\mathbf{c}_{i}=\mathbf{z}_{i} / n_{i}, i=1,2, \ldots, m$. Suppose the contrary. Then there exist rational numbers $\lambda_{1}, \lambda_{2}, \ldots, \lambda_{m}$, not all zero, such that

$$
\lambda_{1} \mathbf{c}_{1}+\lambda_{2} \mathbf{c}_{2}+\cdots+\lambda_{m} \mathbf{c}_{m}=\mathbf{0}
$$

Without loss of generality we may assume that $\lambda_{1}, \lambda_{2}, \ldots, \lambda_{m}$ are integers, with no nontrivial overall common factor. It follows immediately that

$$
\left\{\lambda_{1} \mathbf{c}_{1}+\lambda_{2} \mathbf{c}_{2}+\cdots+\lambda_{m} \mathbf{c}_{m}\right\}=\mathbf{0}
$$

Since the rule is assumed to be nonrepetitive, it follows from Lemma 2.2 that

$$
\lambda_{i} \text { is a multiple of } n_{i}, \quad i=1,2, \ldots, m .
$$

But because the rule is also minimal, it follows from Theorem 4.2 that the numbers $n_{1}, \ldots, n_{m}$ must have a nontrivial common factor, say $a$, and since we have shown that $\lambda_{i}$ is a multiple of $n_{i}$, it now follows that $a$ is a common factor of each of $\lambda_{1}, \ldots, \lambda_{m}$. Since this gives a contradiction, the result is proved.

There follows an important consequence:

COROLLARY 4.4. The rank $m$ of an s-dimensional lattice rule satisfies $1 \leq$ $m \leq s$.

Now we state the main theorem of this section. This is an immediate transcription of Theorem 3.1, but incorporates the result of Theorem 4.3 above.

THEOREM 4.5. An s-dimensional lattice rule $Q$ can be expressed as a nonrepetitive form

$$
Q f=\frac{1}{n_{1} n_{2} \cdots n_{m}} \sum_{j_{1}=1}^{n_{1}} \sum_{j_{2}=1}^{n_{2}} \cdots \sum_{j_{m}=1}^{n_{m}} \bar{f}\left(\frac{j_{1} \mathbf{z}_{1}}{n_{1}}+\frac{j_{2} \mathbf{z}_{2}}{n_{2}}+\cdots+\frac{j_{m} \mathbf{z}_{m}}{n_{m}}\right)
$$


where $m$ (the 'rank') and $n_{1}, n_{2}, \ldots, n_{m}$ (the 'invariants') are uniquely determined natural numbers satisfying $1 \leq m \leq s$ and

$$
n_{i+1} \mid n_{i}, \quad i=1, \ldots, m-1 ; n_{m}>1 \text {. }
$$

The vectors $\mathbf{z}_{1}, \mathbf{z}_{2}, \ldots, \mathbf{z}_{m}$ are linearly independent.

A lattice rule satisfying the conditions of this theorem will be said to be in canonical form.

We now extend slightly the definition of the invariants.

Definition. A lattice rule satisfying the conditions of Theorem 4.5 (and therefore being of rank $m$ ) will be said to have invariants $n_{1}, n_{2}, \ldots, n_{s}$, with $n_{1}, n_{2}, \ldots, n_{m}$ taking the values in (4.7) and $n_{m+1}=n_{m+2}=\cdots=n_{s}=1$.

It is often convenient to suppress the trivial invariants, that is those whose value is 1 . The nomenclature then corresponds precisely to that for the abscissa set $A(Q)$ regarded as a group. Note that the product of the invariants is the number $\nu(Q)$ of abscissas.

COROLlARY 4.6. A quadrature rule which can be expressed as a nonrepetitive form (4.6) with $n_{1}, n_{2}, \ldots, n_{m}$ satisfying (4.7) is a lattice rule with rank $m$ and invariants $n_{1}, n_{2}, \ldots, n_{m}$.

This follows from Theorem 2.1 together with the uniqueness provision of Theorem 4.5.

We conclude with an example. Suppose

$$
Q f=\frac{1}{36} \sum_{j_{1}=1}^{6} \sum_{j_{2}=1}^{6} \bar{f}\left(j_{1} \frac{(5,0,2)}{6}+j_{2} \frac{(4,3,1)}{6}\right) .
$$

The reader will easily verify, by writing out the abscissas, that this form of the rule is repetitive, and that the abscissa set $A(Q)$ contains just 12 points, namely those given in (3.4). Thus, this example has already been analyzed in Section 3. The direct-sum representation (3.6)-(3.8), conforming as it does to Theorem 3.1, corresponds to the expression

$$
Q f=\frac{1}{12} \sum_{j_{1}=1}^{6} \sum_{j_{2}=1}^{2} \bar{f}\left(j_{1} \frac{(1,3,1)}{6}+j_{2} \frac{(0,1,1)}{2}\right),
$$

which is precisely of the canonical form described in Theorem 4.5. This 3-dimensional rule has rank 2 and invariants $6,2,1$; or, more briefly, invariants 6,2 .

5. Rule Projection. Given an arbitrary quadrature rule $Q f$ for the $s$-dimensional cube $C^{s}$,

$$
Q f=\sum_{j=1}^{\nu} w_{j} f\left(x_{j}^{1}, x_{j}^{2}, \ldots, x_{j}^{s}\right),
$$

we define an $s^{\prime}$-dimensional projection $Q^{\prime} f$, with $s^{\prime}<s$, to be any rule obtained by omitting $s-s^{\prime}$ specified components. Most simply, if the last $s-s^{\prime}$ components are omitted, we obtain the principal $s^{\prime}$-dimensional projection of $Q$, namely

$$
Q^{\prime} f=\sum_{j=1}^{\nu} w_{j} f\left(x_{j}^{1}, x_{j}^{2}, \ldots, x_{j}^{s^{\prime}}\right)
$$


which is a quadrature rule (possibly in repetitive form) over the $s^{\prime}$-dimensional cube $C^{s^{\prime}}$.

In the case of a lattice rule, every projected rule has a structure that derives from that of its parent:

THEOREM 5.1. Let $Q$ be an s-dimensional lattice rule having rank $m$ and invariants $n_{1}, n_{2}, \ldots, n_{m}$. Any $s^{\prime}$-dimensional projection $Q^{\prime}$ is a lattice rule having rank $m^{\prime} \leq m$ and invariants $n_{1}^{\prime}, n_{2}^{\prime}, \ldots, n_{m^{\prime}}^{\prime}$, where $n_{i}^{\prime} \mid n_{i}$ for $i=1,2, \ldots, m^{\prime}$.

Proof. The abscissa set $A(Q)$ has a subgroup $H$ consisting of all the abscissas whose only nonzero components (if any) are omitted in the formation of $Q^{\prime}$. In other words, $H$ consists of the abscissas that project onto the origin. Then $A\left(Q^{\prime}\right)$ is isomorphic to the factor group $A(Q) / H$ (see Section 3), and the theorem is an immediate consequence of Theorem 3.4.

COROLlARY 5.2. Let $Q$ be an $N$-point $s$-dimensional lattice rule, and let $Q^{\prime}$ be an $s^{\prime}$-dimensional projection. If $Q^{\prime}$ has $N$ distinct abscissas, then $Q^{\prime}$ has the same rank and invariants as $Q$.

Proof. This follows immediately from Theorem 5.1 and the fact that the product of the invariants of $Q^{\prime}$ is the same as the product of the invariants of $Q$, since both are equal to $N$.

To illustrate rule projection, we return to the 3-dimensional rule (4.9),

$$
Q f=\frac{1}{12} \sum_{j_{1}=1}^{6} \sum_{j_{2}=1}^{2} \bar{f}\left(j_{1} \frac{(1,3,1)}{6}+j_{2} \frac{(0,1,1)}{2}\right)
$$

whose abscissas are listed in (3.4). Being already in the standard form of Theorem 4.5, this rule manifestly has rank 2 and invariants 6,2 .

There are three two-dimensional projections of this rule, into the $x^{1}, x^{2}$ plane, the $x^{1}, x^{3}$ plane, and the $x^{2}, x^{3}$ plane. These are respectively the two-dimensional rules

$$
\begin{aligned}
& Q_{12} f=\frac{1}{12} \sum_{j_{1}=1}^{6} \sum_{j_{2}=1}^{2} \bar{f}\left(j_{1} \frac{(1,3)}{6}+j_{2} \frac{(0,1)}{2}\right), \\
& Q_{13} f=\frac{1}{12} \sum_{j_{1}=1}^{6} \sum_{j_{2}=1}^{2} \bar{f}\left(j_{1} \frac{(1,1)}{6}+j_{2} \frac{(0,1)}{2}\right), \\
& Q_{23} f=\frac{1}{12} \sum_{j_{1}=1}^{6} \sum_{j_{2}=1}^{2} \bar{f}\left(j_{1} \frac{(3,1)}{6}+j_{2} \frac{(1,1)}{2}\right)=\frac{1}{6} \sum_{j=1}^{6} \bar{f}\left(j \frac{(3,1)}{6}\right) .
\end{aligned}
$$

By inspection, the first two are in nonrepetitive form, and so have rank $m=2$ and invariants 6,2 . The third appears first in a repetitive form, but as indicated may be rewritten in nonrepetitive form with rank 1 and invariant 6.

The key to predicting this situation is to examine the list of abscissas (3.4) of $Q$ to see how many project into the origin. In the first two cases, $Q_{12}$ and $Q_{13}$, only one element projects to $(0,0)$. We have then the uninteresting case in Theorem 5.1 in which $H$ is the identity and $A(Q) / H$ is isomorphic to $A(Q)$. In the third case, 
pertaining to $Q_{23}$, inspection of the list (3.4) shows that two elements project to $(0,0)$. These two elements now constitute $H$, i.e.,

$$
H=\left\{(0,0,0),\left(\frac{1}{2}, 0,0\right)\right\},
$$

giving immediately that $A(Q) / H$ is of order 6 . The group $A(Q) / H$ is described in Section 3 and is seen there to be a cyclic group of order 6 . The import of Theorem 5.1 is that the abscissa set $A\left(Q_{23}\right)$ is isomorphic with this group, and so is also a cyclic group. Thus $A\left(Q_{23}\right)$ has rank 1 and invariant 6 , in agreement with the statement above for the rule $Q_{23}$.

6. $n^{s}$-Copy Rules. The $n^{s}$-copy $Q^{(n)}$ of a general quadrature rule

$$
Q f=\sum_{j=1}^{\nu} w_{j} f\left(\boldsymbol{x}_{j}\right)
$$

for the unit cube $C^{s}$ is the rule obtained by subdividing $C^{s}$ into $n^{s}$ cubes each of side $n^{-1}$, and applying a properly scaled version of the rule $Q$ to each smaller cube. Specifically,

$$
Q^{(n)} f=\sum_{k_{1}=0}^{n-1} \sum_{k_{2}=0}^{n-1} \cdots \sum_{k_{s}=0}^{n-1} \sum_{j=1}^{\nu} \frac{w_{j}}{n^{s}} f\left(\frac{\left(k_{1}, k_{2}, \ldots, k_{s}\right)}{n}+\frac{x_{j}}{n}\right) .
$$

In particular, with the aid of (2.5), the $n^{s}$-copy of the lattice rule (2.8) is

$$
Q^{(n)} f=\frac{1}{n^{s} N} \sum_{k_{1}=0}^{n-1} \sum_{k_{2}=0}^{n-1} \cdots \sum_{k_{s}=0}^{n-1} \sum_{j=1}^{N} \bar{f}\left(\frac{\left(k_{1}, k_{2}, \ldots, k_{s}\right)}{n}+\frac{x_{j}}{n}\right) .
$$

THEOREM 6.1. The $n^{s}$-copy of a lattice rule is a lattice rule.

Proof. This follows from the original definition of a lattice rule through an infinite lattice $L$. Defining a lattice $L^{(n)}$ by

$$
x \in L \rightarrow x / n \in L^{(n)},
$$

we find directly that the lattice rule constructed using $L^{(n)}$ is precisely the $n^{s}$-copy rule.

For example, the $n^{s}$-copy of the one-point rule $T_{s}^{(1)} f=\bar{f}(0)$ is the $n^{s}$-product trapezoidal rule $T_{s}^{(n)}$. The product trapezoidal rule plays a central role in the study of $n^{s}$-copy rules. From the definition (2.14) and Corollary 4.6 we have immediately:

LEMMA 6.2. The $n^{s}$-product trapezoidal rule $T_{s}^{(n)}$ is a lattice rule of rank $s$ and invariants $n, n, \ldots, n$.

And from the definition (6.3) of the $n^{s}$-copy $Q^{(n)}$ we have:

LEMMA 6.3. The abscissa set $A\left(Q^{(n)}\right)$ of the $n^{s}$-copy of a lattice rule $Q$ contains the abscissa set $A\left(T_{s}^{(n)}\right)$ as a subgroup.

THEOREM 6.4. The $n^{s}$-copy of a lattice rule is a lattice rule of rank $s$. The $s$ invariants all have $n$ as a common factor.

Proof. It follows from Lemmas 6.3 and 6.2 and from Theorem 3.3 that there are $s$ invariants of $A\left(Q^{(n)}\right)$ all having $n$ as a factor. 
LEMMA 6.5. If the s-dimensional lattice rule $Q$ is expressible in nonrepetitive form as

$$
Q f=\frac{1}{n_{1} n_{2} \cdots n_{s}} \sum_{j_{1}=1}^{n_{1}} \sum_{j_{2}=1}^{n_{2}} \cdots \sum_{j_{s}=1}^{n_{s}} \bar{f}\left(\frac{j_{1} \mathbf{z}_{1}}{n_{1}}+\frac{j_{2} \mathbf{z}_{2}}{n_{2}}+\cdots+\frac{j_{s} \mathbf{z}_{s}}{n_{s}}\right),
$$

where all $n_{i}$ have a factor $k>1$, then $Q$ is the $k^{s}$-copy of the rule

$$
P f=\frac{1}{\nu_{1} \nu_{2} \cdots \nu_{s}} \sum_{r_{1}=1}^{\nu_{1}} \sum_{r_{2}=1}^{\nu_{2}} \cdots \sum_{r_{s}=1}^{\nu_{s}} \bar{f}\left(\frac{r_{1} \mathbf{z}_{1}}{\nu_{1}}+\frac{r_{2} \mathbf{z}_{2}}{\nu_{2}}+\cdots+\frac{r_{s} \mathbf{z}_{s}}{\nu_{s}}\right)
$$

with $\nu_{i}=n_{i} / k, i=1,2, \ldots, s$.

Proof. In the expression (6.5) we may decompose the sum over $j_{i}$ by setting

$$
j_{i}=l_{i} \nu_{i}+r_{i}, \quad i=1,2, \ldots, s,
$$

and summing $l_{i}$ over $0,1, \ldots, k-1$ and $r_{i}$ over $1, \ldots, \nu_{i}$. Thus we obtain

$$
\begin{aligned}
& Q f=\frac{1}{k^{s} \nu_{1} \nu_{2} \cdots} \nu_{s} \\
& \sum_{l_{1}=0}^{k-1} \ldots \sum_{l_{s}=0}^{k-1} \sum_{r_{1}=1}^{\nu_{1}} \ldots \sum_{r_{s}=1}^{\nu_{s}} \\
& \quad \times \bar{f}\left(l_{1} \frac{\mathbf{z}_{1}}{k}+l_{2} \frac{\mathbf{z}_{2}}{k}+\cdots+l_{s} \frac{\mathbf{z}_{s}}{k}+r_{1} \frac{\mathbf{z}_{1}}{k \nu_{1}}+\cdots+r_{s} \frac{\mathbf{z}_{s}}{k \nu_{s}}\right) .
\end{aligned}
$$

Because $Q$ has $n_{1} n_{2} \cdots n_{s}$ distinct abscissas, all the quadrature points in the latter expression must be distinct. In particular, the $k^{s}$. points

$$
\left\{l_{1} \frac{\mathbf{z}_{1}}{k}+\cdots+l_{s} \frac{\mathbf{z}_{s}}{k}\right\}, \quad l_{i}=0,1, \ldots, k-1, i=1, \ldots, s
$$

must be distinct, and so these must be just the abscissas of the $k^{s}$-point product trapezoidal rule $T_{s}^{(k)}$ taken in a different order.

Thus, $Q$ is expressible as

$$
\begin{aligned}
Q f=\frac{1}{k^{s} \nu_{1} \nu_{2} \cdots \nu_{s}} \sum_{k_{1}=0}^{k-1} & \cdots \sum_{k_{s}=0}^{k-1} \sum_{r_{1}=1}^{\nu_{1}} \cdots \sum_{r_{s}=1}^{\nu_{s}} \\
& \times \bar{f}\left(\frac{\left(k_{1}, k_{2}, \ldots, k_{s}\right)}{k}+r_{1} \frac{\mathbf{z}_{1}}{k \nu_{1}}+\cdots+r_{s} \frac{\mathbf{z}_{s}}{k \nu_{s}}\right)
\end{aligned}
$$

which according to (6.3) is just the $k^{s}$-copy of the rule (6.6).

The following theorem is an immediate corollary of this lemma.

THEOREM 6.6. Any s-dimensional lattice rule of rank $s$ having invariants $n_{1}, n_{2}, \ldots, n_{s}$ is the $n_{s}^{s}$-copy of a lattice rule of rank less than $s$ having invariants $n_{1} / n_{s}, n_{2} / n_{s}, \ldots, n_{s} / n_{s}$.

Proof. This follows from choosing $n_{1}, n_{2}, \ldots, n_{s}$ in Lemma 6.5 to be the invariants of $Q$ and setting $k=n_{s}$. In this case we have $n_{i+1} \mid n_{i}$, so all $n_{i}$ have the common factor $k=n_{s}$. This implies $\nu_{i+1} \mid \nu_{i}$ and $\nu_{s}=1$. Since (6.6) is a nonrepetitive form, it follows that $P f$ has invariants $\nu_{1}, \nu_{2}, \ldots, \nu_{s}$; thus the copied rule $P f$ is of rank $m$, where $m$ is the largest value of $t$ for which $\nu_{t}>1$ (or the largest value of $t$ for which $n_{t}>n_{s}$ ).

The following theorem is a converse. 
THEOREM 6.7. The $n^{s}$-copy of an s-dimensional lattice rule $Q$ having invariants $n_{1}, n_{2}, \ldots, n_{s}$ is a lattice rule having invariants $n n_{1}, n n_{2}, \ldots, n n_{s}$.

Proof. From Theorem 6.4 the $n^{s}$-copy of $Q$ has invariants having an overall factor $n$. Suppose these are $n \mu_{1}, n \mu_{2}, \ldots, n \mu_{s}$. Then Lemma 6.5 tells us that $Q$ itself has invariants $\mu_{1}, \mu_{2}, \ldots, \mu_{s}$. But since the invariants of $Q$ are $n_{1}, n_{2}, \ldots, n_{s}$, the theorem follows.

We close by remarking that the converse of Lemma 6.5 is not true. The situation is that when form (6.5) is nonrepetitive, then as we have shown, so is form (6.6), and $Q$ is the $k^{s}$-copy of $P$. But when we start with a form (6.6) which is nonrepetitive and construct (6.5), we may either find $Q f$ to be nonrepetitive and to be the $k^{s}$-copy of $P$, or find $Q f$ to be repetitive and to have an abscissa set $A(Q) \subset A\left(P^{(k)}\right)$.

Acknowledgment. We are grateful to Drs. P. Donovan and D. Hunt of the University of New South Wales for assistance with aspects of the theory of finite abelian groups, and to the Australian Research Grants Scheme and the U. S. Department of Energy for generous financial support.

School of Mathematics

University of New South Wales

Sydney, N.S.W. 2033, Australia

Mathematics and Computer Science Division

Argonne National Laboratory

9700 South Cass Avenue

Argonne, Illinois 60439-4844

1. H. CONROY, "Molecular Schrödinger equation, VIII: A new method for the evaluation of multidimensional integrals," J. Chem Phys., v. 47, 1967, pp. 5307-5318.

2. R. CRANley \& T. N. L. PAtterson, "Randomization of number-theoretic methods for multiple-integration," SIAM J. Numer. Anal., v. 13, 1976, pp. 904-914.

3. M. HALL, JR., The Theory of Groups, Macmillan, New York, 1959.

4. E. HLAWkA, "Zur angenäherten Berechnung mehrfacher Integrale," Monatsh. Math., v. 66, 1962, pp. $140-151$.

5. HUA LOO Keng \& WANg YUAN, Applications of Number Theory to Numerical Analysis, Springer-Verlag, Berlin, Science Press, Beijing, 1981.

6. T. W. Hungerford, Algebra, Springer-Verlag, New York, 1974.

7. P. KEAST, "Optimal parameters for multidimensional integration," SIAM J. Numer. Anal., v. 10,1973, pp. $831-838$.

8. N. M. KOROBOV, "The approximate computation of multiple integrals," Dokl. Akad. Nauk $S S S R$, v. 124, 1959, pp. 1207-1210. (Russian)

9. W. LedermanN, Introduction to the Theory of Finite Groups, Oliver and Boyd, Edinburgh, 1964.

10. H. NiederReiter, "Quasi-Monte Carlo methods and pseudo-random numbers," Bull. Amer. Math. Soc., v. 84, 1978, pp. 957-1041.

11. I. H. SlOAN, "Lattice methods for multiple integration," J. Comput. Appl. Math., v. 12 and 13, 1985, pp. 131-143.

12. I. H. SLOAN \& P. J. KACHOYAN, "Lattice methods for multiple integration: theory, error analysis and examples," SIAM J. Numer. Anal., v. 24, 1987, pp. 116-128.

13. S. K. ZAREMBA, "La méthode des "bons treillis" pour le calcul des intégrales multiples," in Applications of Number Theory to Numerical Analysis (S. K. Zaremba, ed.), Academic Press, London, 1972, pp. 39-116. 